Annuaire suisse de politique de développement

$10 \mid 1991$

Annuaire Suisse - Tiers Monde 1991

\title{
La Suisse face à une éventuelle entrée au FMI et à la Banque Mondiale (BM)
}

\section{Mario Carera}

\section{(2) OpenEdition}

Édition électronique

URL : http://journals.openedition.org/aspd/1380

DOI : $10.4000 /$ aspd. 1380

ISSN : 1663-9669

Éditeur

Institut de hautes études internationales et du développement

\section{Édition imprimée}

Date de publication : 1 janvier 1991

Pagination : 215-218

ISSN : 1660-5934

\section{Référence électronique}

Mario Carera, «La Suisse face à une éventuelle entrée au FMl et à la Banque Mondiale (BM) »,

Annuaire suisse de politique de développement [En ligne], 10 | 1991, mis en ligne le 13 avril 2013, consulté le 08 septembre 2020. URL : http://journals.openedition.org/aspd/1380 ; DOI : https:// doi.org/10.4000/aspd. 1380 


\title{
La Suisse face à une éventuelle entrée au FMI et à la Banque Mondiale (BM)
}

\author{
Mario Carera
}

Du point de vue de la politique de développement, l'éventuelle entrée de la Suisse au FMI et à la Banque Mondiale (BM) doit s'analyser à deux niveaux:

I. l'évolution du rôle de ces deux institutions

II. la politique que la Suisse suivrait en leur sein en cas d'entrée.

I. Dans l'évolution des deux Institutions de Bretton Woods, relevons quelques faits importants:

- L'universalité: créés par 44 nations au lendemain de la dernière guerre, le FMI et la BM sont devenus des institutions universelles (156 pays membres aujourd'hui, avec les récentes adhésions de la Tchécoslovaquie, de la Bulgarie, de la Namibie et de la Mongolie). Si l'on excepte I'URSS, surtout, et l'Albanie, qui ont toutes deux déjà exprimé leur intérêt pour une adhésion, seuls Cuba, la Corée du Nord et la Suisse restent à l'écart. En mai 1990, les pays du tiers monde ont vivement soutenu le doublement des ressources du FMI, contre la position des pays riches (G7) qui se sont limités à $50 \%$.

- Le multilatéralisme: la globalisation des marchés financiers et commerciaux, l'interdépendance des problèmes de développement, d'endettement, d'environnement, la reconstruction économique de l'Est européen requièrent des approches multilatérales. Les institutions de Bretton Woods (et les organisations multilatérales en général) sont l'enjeu de luttes d'influence, reflets de conflits d'intérêts, de rapports de force mondiaux en pleine évolution. L'al- 
ternative, qui n'en est pas une, serait le bilatéralisme ou l'unilatéralisme, l'arme des puissances du Nord (Etats-Unis en Amérique latine, France en Afrique de l'Ouest...).

- Le pouvoir institutionnel au FMI et à la BM n'est pas équitable et doit étre revu. Le droit de vote est réparti en fonction des quotes-parts (cotisations) versées. Les Etats-Unis, à eux seuls, avec $19,1 \%$ des voix, disposent d'une minorité de blocage. Cette répartition des voix en fonction du poids économique des pays membres se retrouve dans les institutions financières internationales, telles les banques régionales de développement, y compris dans la banque européenne de développement créée au printemps 1990 pour faciliter la reconstruction des économies des pays de l'Est, à laquelle la Suisse a adhéré. Une première réforme du droit de vote au FMI devrait partir de la base définie en 1947 (12,5\% comme droit de vote de base + le reste dépendant des quotes-parts), ce qui porterait les droits de vote de base aujourd'hui à $33 \%$, si l'on appliquait le facteur multiplicateur des quotes-parts, introduites depuis.

- Les turbulences monétalres du début des années 70 ont fait éclater le système de la libre convertibilité des monnaies entre elles à taux fixe, conçu à Bretton Woods en juillet 1944. Depuis le début des années 70 , et de façon encore plus marquée avec la crise de l'endettement international dès le début des années 80 , le FMI "gère" les déséquilibres monétaires et de balance des paiements internationaux, plus qu'il ne s'y attaque. Un "nouveau Bretton Woods" devra jeter les bases d'un nouvel ordre financier international (sortant du dollar), d'où résultera un FMI "rénové", institution garante du fonctionnement de ce nouveau système.

- Les programmes d'ajustement structurels (PAS) engagés par le FMI et la BM depuis une décennie dans les pays en voie de développement (pvd) en crise (déséquilibre de la balance des paiements, du budget, forte inflation, ...) et la conditionnalité qui leur est liée, font la part trop belle aux classiques équilibres macro-économiques et doivent intégrer de nouveaux paramètres économiques, sociaux, politiques et écologiques. En effet, si certains pvd sous PAS ont réussi à stabiliser quelque peu l'inflation, à diminuer le déficit extérieur et budgetaire, fort peu sont en voie d'assurer leur développement par une "croissance durable et équitable" (selon l'expression de la BM).

- La BM, surtout, et le FMI ne sont pas des blocs monolithiques. Une évolution est perceptible depuis quelques années, liée à leur róle croissant dans la crise de l'endettement et à l'échec des premières conceptions des PAS:

a) Politique sectorielle

Création, à la BM, de l'unité "dimension sociale de l'ajustement" pour mieux intégrer et cibler l'appui aux populations les plus pauvres dans certaines phases de l'ajustement; création d'une unité "environnement" pour l'Afrique et de façon plus générale, prise de conscience de la nécessité d'une "composante verte" dans la définition des programmes; doublement des prêts de la BM en faveur de l'éducation de base. 


\section{b) Stratégle d'intervention}

Découverte des acteurs de la société civile (syndicats, ONG...), et non pas seulement des Ministères des Finances, du Plan ou de la Banque centrale, dans les Etats concernés et discussions des stratégies "d'accès aux pauvres". En 1989, 50 des 225 projets approuvés par la BM impliquent des ONG. Avec les risques et les contraintes que cela implique, ces soutiens sont appelés à se renforcer de par la nature des problèmes à affronter.

c) Dialogue Institutionnel

Un "World Bank NGO Committee", créé en 1982, regroupant des représentants de la BM et 26 délégués d'ONG du monde entier (en majorité du Sud) poursuit un dialogue critique.

\section{Politique de la Sulsse au FMl et à la Banque Mondiale}

L'analyse de l'évolution des institutions de Bretton Woods prête certes à controverses au sein des ONG suisses et internationales. Dans la perspective d'une adhésion de notre pays, c'est finalement le róle que la Suisse pourrait ou devrait jouer au sein des institutions de Bretton Woods qui est déterminant.

Etant donné le rôle-clé joué par le FMI et la BM dans la crise de l'endettement du tiers monde et la réorganisation des économies des pays du Sud, l'adhésion éventuelle de la Suisse à ces deux institutions dolt s'inscrire dans la continulté de la politique suisse de développement et, en particulier, s'appuyer sur les principes et buts de la loi fédérale sur la coopération au développement et l'aide humanitaire internationale de 1976. Cette loi s'est avérée un bon instrument de référence pour la coopération suisse, ce qui est souligné chaque année par le Comité d'aide au développement (CAD) de l'OCDE.

C'est pourquoi la Communauté de travall SWISSAID/ACTION DE CAREME/PAIN POUR LE PROCHAIN/HELVETAS falt de la référence explicite à cette lol fédérale sur la coopération au développement dans l'arrêté fédéral prévoyant l'adhésion au FMl et la BM la condition de base pour son accord à l'adhésion de la Suisse à ces deux institutions.

La Communauté de travail SWISSAID/ACTION DE CAREME/PAIN POUR LE PROCHAIN/HELVETAS formule en outre 4 points spécifiques devant retenir l'attention de nos autorités:

1) Contenu/principes:

A part le respect des principes et objectifs de la loi sur la coopération, la Suisse doit aussi se prononcer pour une politique visant la réalisation de programmes d'ajustements dans les pays industrialisés et dans leurs relations avec les pvd. "Sans environnement extérieur favorable", en effet, les efforts internes d'ajustement entrepris par de nombreux pvd sont voués à l'échec. FMI èt BM dénoncent régulièrement les distorsions présentes dans 
les économies du Nord (déficit américain, protectionnisme, baisse du prix des matières premières...), sans que des solutions sérieuses - question de poids politique des pays concernés? - ne soient réellement engagées.

2) Le princlpe de transparence dolt étre respecté

Le Conseil fédéral rendra compte au Parlement des politiques suivies par la Suisse au sein des deux institutions; les organisations suisses d'entraide, avocats des intérêts des plus pauvres, seront consultées et informées.

3) Représentation:

Eu égard à son poids financier et afin qu'elle exerce pleinement sa responsabilité internationale, il serait souhaitable que la Suisse obtienne un siège au conseil d'administration du FMI. La représentation de la Suisse à la BM devrait être confiée au Département fédéral des affaires étrangères, vu le caractère "de développement" de cette institution. Au FMI elle doit revenir au Département des finances.

\section{4) Ressources}

Ni la qualité, ni la quantité de l'aide publique suisse ne doivent être réduites par cette adhésion. Au contraire, du point de vue budgétaire, le Conseil fédéral doit s'engager à atteindre l'objectif de $0,5 \%$ du PNB, moyenne des contributions des pays de la $\mathrm{CE}$, comme aide publique au développement. Crédibilité internationale et Eurocompatibilité obligent! Toute augmentation ultérieure de la quote-part de la Suisse au FMI devrait par ailleurs être décidée par le Parlement.

Si ces principes de politique de développement fondent notre politique future dans le cadre du FMI et de la BM, la Suisse renforcerait l'évolution en cours au sein des deux institutions (pays nordiques, conditionnalité verte de certains pays de la $C E$, de certains pvd) et cette adhésion prendrait alors tout son sens: celle d'un petit pays financièrement et économiquement important, désireux de participer de plein pied aux débats de la Communauté internationale. Un pays assumant au fond ses droits civiques Internationaux. Beau défi de politique intérieure aussi pour les ONG suisses! 